\title{
Mendes, Emília \& Ida Lucia Machado (éds). 2010. As emoções no discurso (Campinas, São Paulo : Mercado de Letras)
}

Giani David Silva

\section{(2) OpenEdition}

Journals

Édition électronique

URL : http://journals.openedition.org/aad/1222

DOI : 10.4000/aad.1222

ISSN : $1565-8961$

Éditeur

Université de Tel-Aviv

Référence électronique

Giani David Silva, « Mendes, Emília \& Ida Lucia Machado (éds). 2010. As emoções no discurso

(Campinas, São Paulo : Mercado de Letras) », Argumentation et Analyse du Discours [En ligne], 7| 2011, mis en ligne le 15 octobre 2011, consulté le 21 septembre 2020. URL : http://journals.openedition.org/ aad/1222 ; DOl : https://doi.org/10.4000/aad.1222

Ce document a été généré automatiquement le 21 septembre 2020.

\section{(i) $९$}

Argumentation \& analyse du discours est mis à disposition selon les termes de la licence Creative Commons Attribution - Pas d'Utilisation Commerciale - Pas de Modification 4.0 International. 


\title{
Mendes, Emília \& Ida Lucia Machado (éds). 2010. As emoções no discurso (Campinas, São Paulo : Mercado de Letras)
}

\author{
Giani David Silva
}

\section{RÉFÉRENCE}

Mendes, Emília \& Ida Lucia Machado (éds). 2010. As emoções no discurso (Campinas, São Paulo : Mercado de Letras), 245 pages, ISBN : 9788575911266

1 Logos, ethos, pathos: conçue dans le cadre d'une rhétorique de l'argumentation, la triade aristotelicienne reste toujours d'actualité dans l'approche préconisée par les tendances de l'analyse du discours qui ont comme principe de prendre en compte le dispositif d'énonciation dans son ancrage sociohistorique. Le logos, on le sait, se rapporte à la dimension raisonnable de l'argumentation, dimension responsable de la cohérence logique des arguments ; l'ethos correspond à l'image de soi que l'orateur produit par son discours et dans son énonciation, car ses façons de dire sont plus révélatrices que ce qu'il dit sur sa propre personne; le pathos, enfin, tente de faire naitre dans l'auditoire des états émotionnels favorables à ce que propose l'orateur. Il faut souligner que les frontières entre raison et émotion sont floues, surtout dans les mises en scène langagières qui mobilisent des stratégies au profit d'un objectif d'influence inscrit dans un contexte social particulier. Plus on avance dans la compréhension de l'un des éléments de la triade rhétorique, plus on découvre ses rapports avec les autres et les effets de sens produits par leur inévitable articulation.

2 Si l'ouvrage brésilien intitulé As emoções no discurso a choisi d'aborder le pathos, c'est dans la mesure où - il faut bien le reconnaître - on manque encore d'une approche des émotions capable de prendre en compte la dynamique intersubjective et les aspects 
ritualisés des interactions verbales. Le premier volume de cette collection (2007), avait privilégié les méthodologies et les instruments d'analyse, préoccupation d'autant plus légitime que les études discursives ne disposent pas d'instruments d'analyse déterminés a priori pour étudier le pathos. Dans le volume 2, l'accent est mis sur la diversité des pratiques langagières dans un effort, peut-être inconscient, de capter le fonctionnement global des émotions dans les discours les plus divers en montrant que les émotions font partie du quotidien, même si l'époque contemporaine appelle l'individu à respecter l'objectivité et les normes du raisonnable. Parmi les réflexions linguistiques présentées dans cet ouvrage, la littérature, l'anthropologie, la sémiotique et la communication sociale ont leur mot à dire, un mot qui entre toujours en résonance avec ce qui a été déjà dit, de la rhétorique jusqu'à nos jours, en accord avec la maxime dialogique proposée par Bakhtine.

3 En ouverture, Patrick Charaudeau propose une perspective d'étude des émotions qui s'inscrit dans le cadre de sa théorie sémiolinguistique, une approche discursive centrée sur l'action communicative du langage. Il montre ainsi comment le dispositif communicatif joue un rôle central dans le fonctionnement pathémique au sein des diverses contraintes et stratégies du discours. Dans une autre perspective, celle des études sur l'argumentation qui caractérise ses travaux, Christian Plantin présente une topique des émotions dans laquelle il met en relief une procédure d'analyse des émotions fondée sur des éléments linguistiques. Il exploite les différents niveaux de l'argumentation, ainsi comprise dans toute sa profondeur.

4 Maria Aparecida Lino Pauliukonis reprend le concept de topos pour traiter des effets pathémiques à partir d'une étude des textes publicitaires brésiliens. Ce matériau permet d'identifier la construction des arguments par le biais des valeurs partagées par une communauté discursive. Dans ce même univers des savoirs figés, Dylia LysardoDias traite des proverbes auxquels elle attribue une dimension pathémique 'naturelle'. De par son caractère de savoir collectivement partagé, les proverbes imposent une interaction sujet-société de façon a favoriser un sentiment et une cohésion de groupe.

5 Ana Maria Clark Peres, quant à elle, se penche sur une proposition de stylistique à partir de Charles Bally pour réfléchir sur les émotions dans la littérature. Elle analyse différents fragments tirés des œuvres de l'écrivaine brésilienne Clarice Lispector, toujours attentive aux rapports entre l'expressivité et le style. João Bosco Cabral dos Santos, comme Beatriz dos Santos Feres, examine l'univers de la lecture et ses rapports pathémiques. Santos se tourne vers l'espace de la mémoire par le biais des entretiens des sujets-lecteurs-scripteurs; il considère les articulations entre savoir, émotion et mémoire. Feres, pour sa part, se consacre aux textes poétiques dans le contexte de la salle de classe et explique la compétence de lecture en la reliant au sentiment et aux sensations.

Adélia Barroso Fernandes consacre son texte à l'étude du discours jounalistique, en utilisant les propostions de la théorie sémiolinguistique. Elle articule l'instance de production et de réception à partir de l'analyse de deux textes d'un même journal brésilien de façon à repérer les effets de dramatisation produits dans ces deux pôles. Cette même thématique de la dramatisation est abordée par Mônica Santos de Souza Melo, elle aussi intéressée par le domaine médiatique : elle nous offre une étude des procédures de spectacularisation et de captation dans des récits portant sur un accident aérien qui a eu lieu au Brésil le 17 juin 2007. 
7 Renata Aparecida Toledo construit une analyse sémiolinguistique d'une chronique littéraire en utilisant la formulation des modes d'organisation discursive proposée par Patrick Charaudeau. L'objet d'étude est une chronique qui thématise le texte en prenant comme point de départ l'interaction entre locuteur et lecteur et en observant le rôle des effets d'affectivité et même de complicité qui se tissent entre ces deux sujets.

Le travail de Thaïs Machado Borges porte sur les «telenovelas » brésiliennes, produit d'exportation qui transmet à l'étranger un univers d'émotions compris selon les normes d'une culture particulière. Borges montre comment ces feuilletons exploitent le domaine des émotions pour capter et séduire le public, en particulier dans leur traitement de l'amour. L'amour est également présent dans l'article de Claudia Natividade et Sônia Pimenta, qui explorent l'univers masculin-féminin à partir d'une lecture sémiotique des émotions. Elles développent une analyse de la communication orale entre adolescents et adultes en prenant pour objet des séquences de cet échange.

9 Le genre épistolaire, lui aussi doté d'un fort impact émotionnel, apparaît dans le volume à travers le travail de Jürgen Siess : il dévoile la polyphonie qui se dégage du discours d'un locuteur qui s'adresse à deux allocutaires sur deux plan différents, en l'occurrence la locutrice des Lettres de Mistress Fanni Butlerd de Marie-Jeanne Riccoboni.

Dans sa diversité d'objets et de parcours analytique, cet ouvrage met en évidence la productivité des recherches qui portent sur le champ des émotions et montre en quoi elles peuvent contribuer à mieux comprendre le fonctionnement du discours. L'interdisciplinarité, déjà présente dans le volume 1 , manifeste dans ce deuxième volume l'importance d'élargir le champ d'investigation pour réfléchir sur les rapports langue-sujets-societé de façon à dégager la dimension culturelle et ethique de la discursivité des émotions. Tels sont en effet les enjeux des efforts déployés pour repérer et analyser les modes d'inscription de l'affectivité dans les discours.

\section{AUTEURS}

\section{GIANI DAVID SILVA}

Centro Federal de Educação Tecnológica de Minas Gerais 